\title{
Impulsive Discharges in Water: Acoustic and Hydrodynamic Parameters
}

\author{
Ying Sun, Igor Timoshkin, Senior Member, IEEE, Martin Given, Member, IEEE, Mark P. Wilson, \\ Member, IEEE, Tao Wang, Scott MacGregor, Senior Member, IEEE, Nelly Bonifaci
}

\begin{abstract}
Underwater spark discharges are used in multiple practical applications including plasma closing switches, water treatment, plasma channel drilling and mineral processing, waste recycling, treatment of metals, and medical lithotripsy. Spark discharges in water have been studied for several decades, however, despite significant research efforts and progress in this area, further investigation into the efficiency of plasma-acoustic sources and their optimisation is required in order to expand their practical application. This paper is aimed at investigation of the electrical and hydrodynamic parameters of underwater plasma-generated cavities, including plasma resistance, energy delivered into the plasma cavity, period of cavity oscillations and characteristics of pressure impulses. Different energy levels, breakdown voltages and gap distances were used in the present study to allow systematic analysis of these electrical and hydrodynamic parameters. Empirical scaling relations which link the maximum acoustic pressure and the period of cavity oscillations with the energisation parameters and the resistance of plasma have been obtained. These empirical functions can be used for optimisation of the plasma-acoustic sources and for tailoring their parameters for specific practical applications.
\end{abstract}

Index Terms-Underwater spark discharges, plasma resistance, hydrodynamic parameters of period of cavity, acoustic impulses

\section{INTRODUCTION}

$\mathrm{I}^{\mathrm{N}}$ $\mathrm{N}$ recent years, significant research efforts have been focused on investigation of high-voltage spark discharges in water. Such discharges have found practical application in different technological areas, including water-filled plasma closing switches used in high-voltage, pulsed-power systems [1], medical lithotripters [2], minerals engineering [3], recycling of solid materials $[4,5,6]$, in water cleaning and remediation operations, and water-well cleaning operations [7]. An important area of potential practical applications of underwater spark discharges is generation of wide-band ultrasound impulses $[8,9]$. Underwater, high-voltage spark discharges

This manuscript was submitted on 21 December 2015, in final form on 20 June 2016. Financial support to Dr Bonifaci was provided by The Royal Academy of Engineering, Dr Bonifaci was visiting the University of Strathclyde as the RAEng Distinguished Fellow

Y. Sun I. Timoshkin, M. Given, M.P. Wilson, T. Wang, S. MacGregor are with the High Voltage Technologies Group, Department of Electronic and Electrical Engineering, University of Straclyde, Glasgow, G1 1XW, UK (E-mail: ying.sun.2013@uni.strath.ac.uk; igor.timoshkin@strath.ac.uk; m.given@strath.ac.uk; mark.p.wilson@strath.ac.uk; tao.wang@strath.ac.uk; barbara.stewart@strath.ac.uk).

N. Bonifaci is with CNRS, G2E Laboratory, Grenoble, France. (E-mail: nelly.bonifaci@g2elab.grenoble-inp.fr)

Digital Object Identifier 10.1109/TPS.2016.2583066 have advantages over traditional methods of generation of pressure impulses, as they provide better controllability and higher reproducibility. The spark-discharge method is also environmentally friendly, as no chemical explosives are used in the plasma-acoustic process. In order to optimise the process of generating acoustic impulses via spark plasma discharges in water, and to tailor their parameters to satisfy specific practical applications, it is important to investigate the characteristics of the acoustic impulses produced under various energisation conditions.

By applying high-voltage impulses with a suitable magnitude, duration and rise-time to a pair of water-immersed electrodes, at least one of which is a point electrode, a highly non-uniform electrical field facilitates electrical breakdown. The breakdown is initiated by the formation of gaseous, pre-breakdown bubble(s)/cavity(ies), generated by a combination of different processes, including Joule heating of the water [10], electron emission, and electro-mechanical rupture of water in the vicinity of the high-potential electrode [11]. The electric field inside this cavity becomes higher than the field in the surrounding water, due to the low permittivity of the gas within the cavity. Once the critical threshold field is exceeded, ionization processes are triggered inside the cavity, resulting in the development of the initial discharge, primarily across the gas/water interface [12]. Plasma streamers then continue to form, progressing through the bulk of the water towards the opposite electrode. The pre-breakdown processes in water stressed with short-duration, high-voltage impulses, including the formation of pre-breakdown gas bubbles and the development of streamers, have been studied in [13 - 17] and other works.

When one of the plasma streamers bridges the inter-electrode gap, the conductive plasma channel closes the circuit, leading to a rapid deposition of energy into the plasma channel (post-breakdown stage). Initially, the plasma resistance is high, due to the relatively-low temperature of the plasma in the channel and its small initial diameter. However, with the establishment of the conductive plasma path and injection of energy into the conductive channel, the plasma inside this channel becomes thermalised and the channel becomes filled with a hot, pressurised, and highly-conductive ionized gas. As the temperature inside the channel rises, the plasma resistance drops rapidly to its minimum value, which is usually in the range between hundreds of $\mathrm{m} \Omega$ and a few $\Omega$ [18]. The post-breakdown cavity rapidly expands into the surrounding water, generating a primary acoustic pressure impulse. The velocity of this pressure impulse rapidly becomes equal to the speed of sound in water. The cavity continues to expand until it 
reaches its maximum size (the gas pressure inside the cavity is minimum at this moment) and then collapses - due to the external hydrostatic pressure - to its minimum size, emitting a secondary acoustic pressure impulse. Several expansion/collapse cycles can potentially be observed [19]. A detailed analysis of the dynamics of the type of post-breakdown plasma/gas cavities formed by spark discharges in water is presented in [20,21]. A hydrodynamic model of the post-breakdown cavity which takes into account the equation of state of water is described in [20]. The development of spark-driven acoustic impulses in water is discussed in [21], considering different electrode topologies: a spherical topology, which is appropriate to shorter inter-electrode gaps; and a cylindrical topology, which can be used for analysis of the acoustic emission from longer, wire-guided discharges.

However, despite a substantial number of publications in the field of plasma discharges in water, the functional behaviour of the acoustic impulses generated by plasma cavities and its link with the hydrodynamic parameters of the cavity and electrical parameters of the circuit are not fully understood, and require further experimental investigation. This paper details an experimental study of the development of plasma-generated, post-breakdown, gaseous cavities in tap water, and analysis of the acoustic impulses produced by such cavities: the dependency of the peak acoustic pressure and the period of cavity oscillations on the electrical energy available in the discharge, the energy-storage capacitance, the breakdown voltage, and the inter-electrode distance. This investigation will help in furthering the understanding of the hydrodynamic behaviour of spark-generated, underwater plasma cavities: the efficiency of production of acoustic impulses and its dependency on the parameters of the pulsed-power driving circuit. The obtained results can be used in the design and development of different plasma-acoustic systems, with parameters tailored for a wide range of practical applications.

\section{EXPERIMENTAL SETUP}

The test system developed for the present study consists of a water tank with dimensions of $2 \mathrm{~m}$ (length) $\times 1 \mathrm{~m}$ (width) $\times$ $1 \mathrm{~m}$ (depth), where spark discharges were generated; a high-voltage, pulsed-power driving circuit, generating negative high-voltage impulses with peak magnitude up to $60 \mathrm{kV}$; and diagnostic devices (high-voltage, high-current and acoustic probes, and digitising oscilloscopes). The pulsed-power system is based on capacitive energy storage: a high-voltage capacitor, or capacitors (Maxwell Laboratories Ltd, USA) connected in parallel when higher energy was required. Three different capacitor combinations were used: two $80 \mathrm{nF}$ capacitors in parallel; one $280 \mathrm{nF}$ capacitor; and two $280 \mathrm{nF}$ capacitors in parallel (these are the nominal capacitance values provided by the manufacturer). Accurate measurements of the capacitances were conducted using a VideoBridge 2160 (ES1-2160, Electro Scientific Instruments), and it was established that these three combinations provided the following values of capacitance: $155 \mathrm{nF}, 266 \mathrm{nF}$ and $533 \mathrm{nF}$. The capacitors were charged by a DC power supply (Glassman High Voltage, series EH, $60 \mathrm{kV}$ ) through a $0.5 \mathrm{M} \Omega$ charging resistor. The system was operated in a single-shot regime, high-voltage impulses being generated by closing a triggered spark-gap switch.
The high-voltage and ground electrodes were located in the middle of the water tank, $\sim 500 \mathrm{~mm}$ below the water surface. Both electrodes were conical and made of stainless-steel, and five different inter-electrode distances were used in this study: $5 \mathrm{~mm}, 7 \mathrm{~mm}, 10 \mathrm{~mm}, 12 \mathrm{~mm}$, and $15 \mathrm{~mm}$.

The voltage and current waveforms were monitored using a NorthStar PVM-5 voltage probe (1000:1 division ratio, $80 \mathrm{MHz}$ bandwidth) and a Samtech Ltd $\mathrm{DE}(\mathrm{CP})-01$ resistive, low-inductance, current shunt $(29.6 \mathrm{~m} \Omega$ resistance). The voltage and current waveforms were recorded using a Tektronix TDS2024 digitising oscilloscope (200 MHz bandwidth, 2 GSamples/s sampling rate). A piezo-electric acoustic transducer, VP1093 Pinducer (Valpey Fisher), was used to obtain the acoustic impulses generated by the spark discharges. This probe was aligned with the mid-point of the inter-electrode gap, and was located $500 \mathrm{~mm}$ away from the spark-discharge source. The Pinducer probe was connected directly to a $1 \mathrm{M} \Omega$ input of a Tektronix TDS3054B digitising oscilloscope, with a bandwidth of $500 \mathrm{MHz}$ and a sampling rate of 5 GSamples/s, in order to monitor the acoustic waveforms (assuming proportionality between voltage signal and acoustic pressure). A schematic diagram of the setup is shown in Fig. 1.

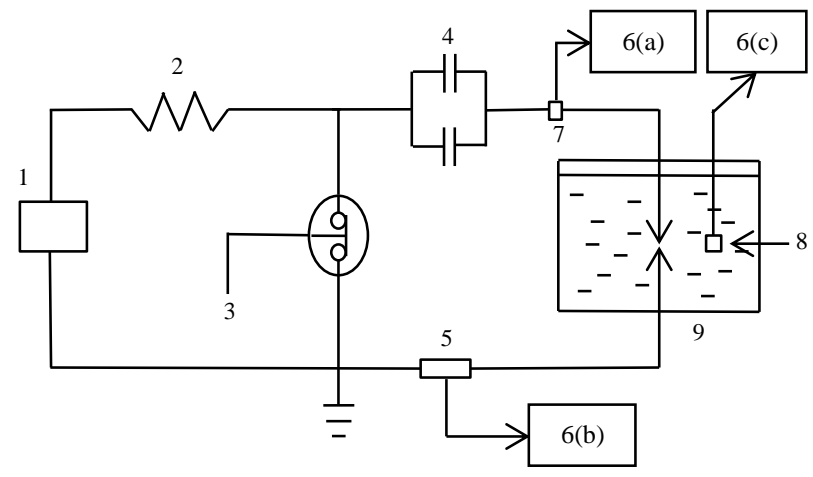

Fig. 1. Schematic diagram of the experimental setup; 1, DC power supply; 2, charging resistor; 3 , spark-gap switch; 4, capacitor bank; 5, current shunt; 6(a), digitising oscilloscope for voltage monitoring; 6(b), digitising oscilloscope for current monitoring; 6(c), digitising oscilloscope for acoustic emission monitoring; 7, voltage probe; 8 , acoustic probe; 9 , water tank.

\section{RESULTS AND ANALYSIS}

\section{A. Electrical and acoustic waveforms}

Spark discharges were generated in tap water (with conductivity of $0.05 \mu \mathrm{S} / \mathrm{cm}$ ) at room temperature, using the pulsed-power circuit shown in Fig. 1. The nominal charging voltage was varied in the range from $20 \mathrm{kV}$ to $35 \mathrm{kV}$ in $5 \mathrm{kV}$ steps. As described in Section II, three values of energy-storage capacitance were used: $155 \mathrm{nF}, 266 \mathrm{nF}$ and $533 \mathrm{nF}$. The inter-electrode gap was varied in the range from $5 \mathrm{~mm}$ to $15 \mathrm{~mm}$. The transient voltage and current waveforms and the corresponding acoustic waveforms generated by plasma discharges were obtained using the diagnostic devices described in Section II. Fig. 2 shows typical voltage and current waveforms obtained following breakdown of a $10 \mathrm{~mm}$ gap.

The voltage and current waveforms obtained in this work are similar in form to the waveforms presented in [18]. The 
decaying current and voltage oscillations indicate that the electrical behaviour of the pulsed-power system and the discharge channel can be described by under-damped sinusoidal oscillations, which are typical for an $R-L-C$ circuit with a constant resistance. The validity of such an assumption has been verified in $[18,19]$. In this model, the current is governed by:

$$
I(t)=I_{0} \exp (-\alpha t) \sin (\omega t)
$$

where

$$
I_{0}=V_{b r} C\left(\frac{\alpha^{2}}{\omega}+\omega\right), \omega=\left(\frac{1}{L C}-\alpha^{2}\right)^{1 / 2}, \quad \alpha=\frac{R}{2 L}
$$

In (1) - (2), $I_{0}$ is the current constant, which is close in value to the maximum current; $V_{b r}$ is the actual breakdown voltage as shown in Fig. 2(a); $L$ and $C$ are the total inductance and capacitance of the circuit, respectively; $R$ is the total resistance; $\alpha$ is the damping factor; and $\omega$ is the angular velocity of the current oscillations.

According to $[22,23]$, the main obstacles to direct measurement of the dynamic plasma resistance are the transient behaviour of the voltage and current in the circuit, and significant reactive components in the plasma-channel impedance. However, the constant resistance approach provides good agreement between the modelled and experimentally-obtained hydrodynamic parameters as presented in [18]. This approach has been validated in the present study by assuming constant resistance and matching the experimental current waveforms with the analytical waveforms calculated using (1), as shown in Fig. 2(b). The analysis presented in this paper is therefore based on the assumption of constant resistance of the plasma channel.
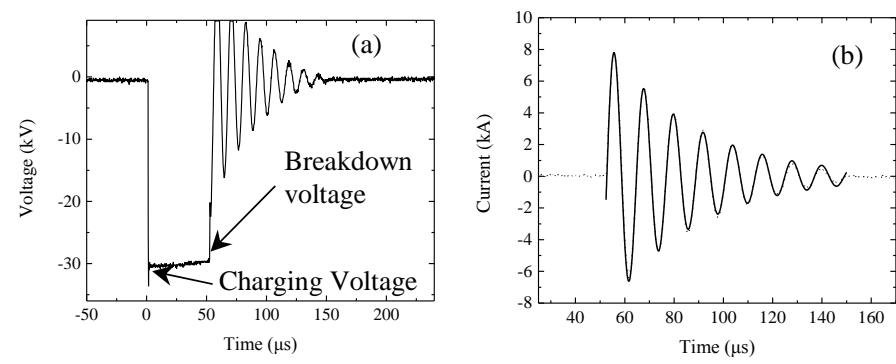

Fig. 2. (a) Voltage and (b) current waveforms for spark discharge with the following parameters: $10 \mathrm{~mm}$ gap, $533 \mathrm{nF}$ energy-storage capacitance, $29.8 \mathrm{kV}$ breakdown voltage ( $30 \mathrm{kV}$ nominal charging voltage). The dotted line in (b) is the experimental $I(t)$, and the solid line is the analytical fitting by (1).

The circuit parameters (inductance, total resistance and constant plasma resistance) can be obtained using the experimental current waveforms and (1) - (2). The constant resistance of the spark plasma, $R_{\text {plasma }}$, can be obtained, using (3), as the difference between the total resistance of the circuit, $R_{\text {total }}$, and the resistance of the circuit without an underwater plasma channel, $R_{\text {circuit }}$ (obtained experimentally under short-circuit load conditions for similar values of peak current):

$$
R_{\text {plasma }}=R_{\text {total }}-R_{\text {circuit }}
$$

The acoustic waveforms generated upon electrical breakdown between the electrodes were monitored using a Pinducer acoustic sensor and a separate digitizing oscilloscope. The characteristics of the acoustic signals were captured using two different oscilloscope time-base settings: one with a time-base of $200 \mathrm{~ns} /$ division to resolve the primary acoustic impulses generated by the expanding plasma cavity; and one with a time-base of $1000 \mu \mathrm{s} /$ division to monitor the complete period of cavity oscillations, by registering the two acoustic impulses produced by the expansion and collapse of a cavity. Fig. 3 shows example acoustic waveforms generated upon breakdown of a $15 \mathrm{~mm}$ water gap. Fig. 3(a) demonstrates the primary acoustic impulse generated by the expanding plasma cavity (shorter timescale), and Fig. 3(b) demonstrates two acoustic signals generated during expansion and collapse of the plasma cavity (longer timescale). The time interval between the primary and secondary acoustic signals is the period of the cavity's oscillation.
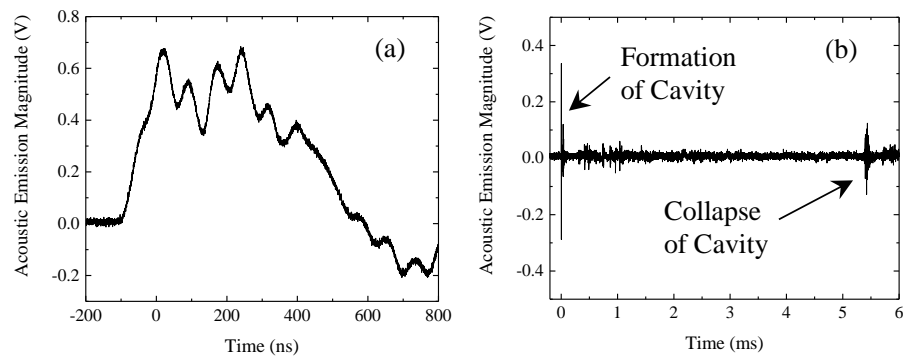

Fig. 3. Acoustic signals generated by the transient underwater plasma cavity. (a), Primary signal due to cavity expansion; (b) primary and secondary signals generated by expanding and collapsing cavity ( $5 \mathrm{~mm}$ gap, $34.4 \mathrm{kV}$ breakdown voltage, $266 \mathrm{nF}$ energy-storage capacitance).

The output transient voltage signal from the ultrasound transducer is proportional to the actual profile of the acoustic signal. However, the calibration coefficients for the acoustic sensor were not available from the manufacturer; therefore, the relative magnitudes of the acoustic signals in Volts were used in the present work. The magnitude of the first acoustic peak (Fig. 3(a)) was obtained and plotted as a function of different circuit parameters, representing the functional behaviour of the maximum pressure in the acoustic wave.

Fig. 3(b) shows two acoustic impulses generated by the same cavity during its expansion and collapse cycle. These signals were registered over a significantly longer time interval, $\sim 4 \mathrm{~ms}$, and they allow the total period of the cavity expansion-collapse cycle to be obtained. The lower-voltage peaks between the two major acoustic spikes represent reflections of the acoustic signal from the water-tank walls and the air/water surface. This longer observation timescale did not allow measurements of the magnitudes of the main peaks to be performed accurately, due to limitations in the digital sampling rate.

\section{B. Analysis and discussion of the experimental results}

\section{1) Period of cavity oscillation}

After completion of the initial, pre-breakdown stage of the discharge, the plasma-filled, post-breakdown cavity expands 
with emission of the primary acoustic impulse, and then collapses under the external hydrostatic pressure. The maximum radius of the cavity and its period of oscillation depend on the energy deposited into this cavity in the transient, post-breakdown process. The cavity's dynamics (its radius as a function of time) are governed by the Rayleigh-Plesset equation [24], which can be integrated assuming that the viscosity and surface tension of the water can be neglected. Thus, an analytical link between the period of the cavity's oscillation, $T_{\text {cavity }}$, and its maximum radius, $R_{\max }$, can be established for the ideal (no energy losses) case:

$$
T_{\text {cavity }}=1.83 R_{\max }\left(\rho_{0} / p_{\infty}\right)^{1 / 2}
$$

where $\rho_{0}$ is the density of water, and $p_{\infty}$ is the ambient hydrostatic pressure.

The electrical energy released in the breakdown channel, $W_{c h}$, is converted in part into thermal plasma energy, $W_{t}$, resulting in cavity expansion:

$$
W_{t}=(4 \pi / 3) R_{\max }^{3} p_{\infty}
$$

The combination of (4) and (5) leads to the following relation between the period of the cavity's oscillations and the thermal plasma energy:

$$
T_{\text {cavity }}=1.14 \sqrt{\rho_{0}} W_{t}^{1 / 3} p_{\infty}^{-5 / 6}
$$

As a portion, $\gamma$, of the total electrical energy released in the plasma channel, $W_{c h}$, is converted into thermal energy in the plasma, $W_{t}$, the link between these energies can be expressed as:

$$
\gamma W_{c h}=W_{t}
$$

The electrical energy, $W_{c h}$, is also proportional to the energy stored in the capacitor bank of the pulsed-power system before breakdown:

$$
W_{c h} \propto C V_{b r}^{2} / 2
$$

Therefore, the period of the cavity's oscillations is proportional to the breakdown voltage to the power $2 / 3$, and to the capacitance to the power 1/3:

$$
T_{\text {cavity }} \propto V_{b r}^{2 / 3} C^{1 / 3} \rho_{0}^{1 / 2} p_{\infty}^{-5 / 6}
$$

In the case of known values of capacitance, fluid density and hydrostatic pressure, a proportionality coefficient, $m$, between $T_{\text {cavity }}$ and $V_{b r}$ can be introduced:

$$
T_{\text {cavity }}=m V_{b r}^{2 / 3}
$$
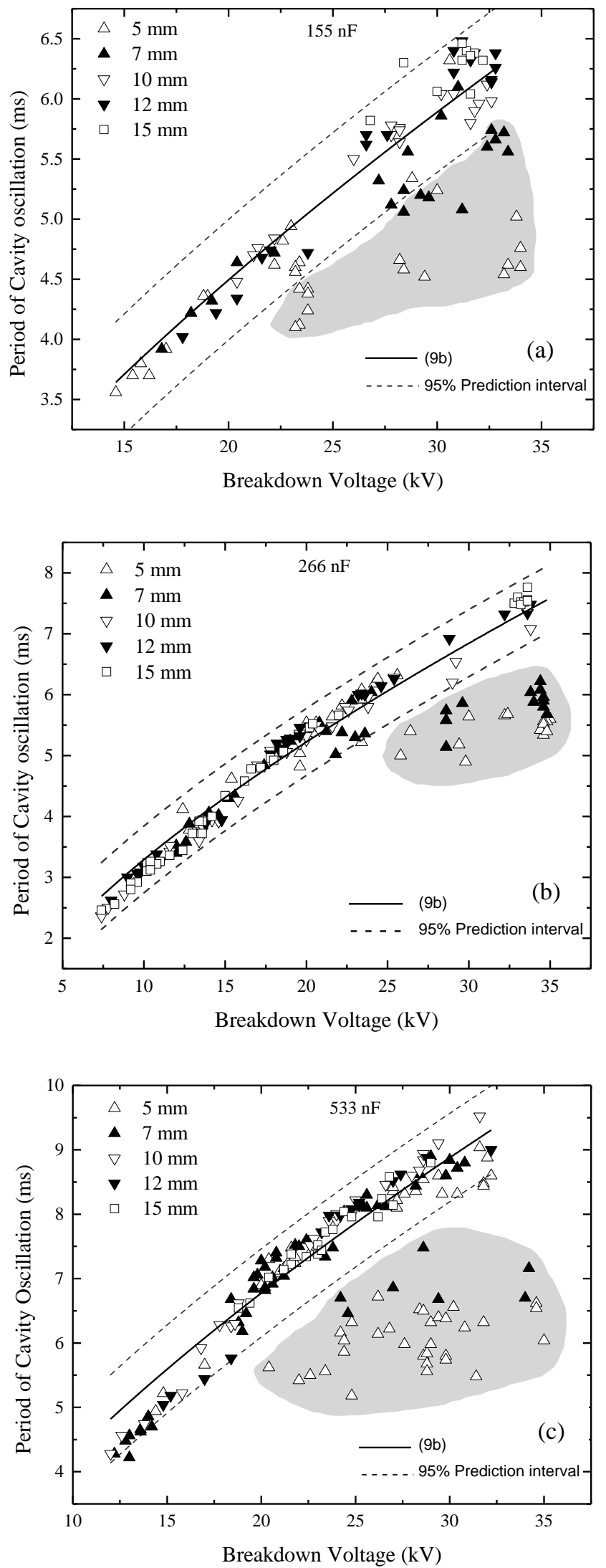

Fig. 4. Period of cavity oscillation as a function of the breakdown voltage for (a) $155 \mathrm{nF}$, (b) $266 \mathrm{nF}$ and (c) $533 \mathrm{nF}$, with the fitting lines given by (9b). The dashed lines show the upper and lower boundaries of the $95 \%$ prediction 
interval. The grey areas indicate those data points where $R_{\text {plasma }}<R_{\text {circuit. }}$

A systematic experimental study of the period of the cavity's oscillation as a function of the breakdown voltage was conducted using five different inter-electrode gaps: $5 \mathrm{~mm}$, $7 \mathrm{~mm}, 10 \mathrm{~mm}, 12 \mathrm{~mm}$, and $15 \mathrm{~mm}$. Three energy-storage capacitances were used in these tests: $155 \mathrm{nF}, 266 \mathrm{nF}$ and $533 \mathrm{nF}$. The results of these measurements (the period of cavity oscillations as a function of breakdown voltage) are shown in Fig. 4, with each data point representing an individual breakdown event. It can be seen that the period of cavity oscillations increases with an increase in the breakdown voltage. However, each graph in Fig. 4 shows that, together with the main increasing trend in the period of oscillations, there are outlying clusters of data points which sit below the lower boundary lines of the $95 \%$ prediction intervals for breakdown voltages of $\sim 20 \mathrm{kV}$ and above. These clusters, highlighted in grey in Fig. 4, are comprised of data points obtained for shorter inter-electrode gaps $(5 \mathrm{~mm}$ and $7 \mathrm{~mm}$ ). Therefore, for these shorter gaps, an increase in the breakdown voltage results in a limited increase in the energy delivered into the plasma channel, despite the higher total energy available in the discharge. The plasma resistance affects the fraction of the stored energy - described by $\gamma$ in (7) - which is delivered into the spark cavity; this resistance has been obtained for all discharges and will be discussed in Section III-B 3). The plasma resistance for all data points located in the outlying grey clusters in Fig. 4 is lower than the circuit resistance, $R_{\text {plasma }}<R_{\text {circuit }}$. Therefore, the deviation of $T_{\text {cavity }}$ from the main trend for shorter gaps and higher breakdown voltages can be explained by the decrease in the efficiency of conversion of electrical energy $\left(C V_{b r}{ }^{2} / 2\right)$ into the thermal energy of the plasma, due to the change in the ratio between the plasma resistance and the remaining resistance of the pulsed-power circuit. This ratio reduces for higher voltages and shorter gaps.

Equation (9b) has been used to fit the experimental data for the period of the cavity oscillations, $T_{\text {cavity }}$ (using data points located within the $95 \%$ prediction intervals in Fig. 4). The fitting procedure was implemented using Origin Pro 9 software. The proportionality coefficient, $m$, was used as a free fitting parameter, and the breakdown voltage, $V_{b r}$, was in $\mathrm{kV}$. The fitting lines obtained by (9b) are shown as solid lines in Fig. 4, while the dashed lines represent the lower and upper $95 \%$ prediction intervals. Numerical values of the fitting parameter, $m$, are given in TABLE I for all three values of capacitance used in these tests.

TABLE I

FITTING PARAMETER $m$ FOR $(9 \mathrm{~b})$

\begin{tabular}{|c|c|c|c|}
\hline$C$ & $155 \mathrm{nF}$ & $266 \mathrm{nF}$ & $533 \mathrm{nF}$ \\
\hline $\begin{array}{c}m \\
95 \% \text { confidence } \\
\text { interval }\end{array}$ & $\begin{array}{c}0.61 \\
(0.61-0.62)\end{array}$ & $\begin{array}{c}0.71 \\
(0.70-0.72)\end{array}$ & $\begin{array}{c}0.92 \\
(0.91-0.93)\end{array}$ \\
\hline
\end{tabular}

From (9a) and (9b), the coefficient $m$ should be proportional to the capacitance to the power 1/3: $m \propto C^{1 / 3}$. A non-linear fit of the data in TABLE I led to the relationship $m \propto C^{0.34}$, which is in good agreement with the expected exponent value of 1/3.

Analysis of the graphs shown in Fig. 4 leads to the following conclusions. For breakdowns where the plasma resistance is greater than the resistance of the driving circuit, the behaviour of $T_{\text {cavity }}$ with varying breakdown voltage follows that predicted by the scaling relationship (9b) for all tested inter-electrode gaps. The coefficient $m$ increases with an increase in the energy-storage capacitance. For shorter gaps and higher breakdown voltages, where the plasma-channel resistance is less than the resistance of the driving circuit, the efficiency of conversion of electrical energy into thermal plasma energy is reduced, resulting in the clusters of data points which fall below the lower boundary lines of the $95 \%$ prediction intervals based on $(9 b)$.

\section{2) Magnitude of acoustic impulses as a function of $V_{b r}$}

For practical development and application of plasma-acoustic sources, it is important to establish a relationship between the breakdown voltage of the water gap and the peak pressure in the primary acoustic impulse radiated by the expanding plasma cavity. This peak pressure is a function of the radius of the cavity, its expansion velocity and its acceleration. The hydrodynamic model described in [18] allows the peak pressure to be obtained as a function of the pulsed-power circuit parameters and plasma resistance. However, the use of phenomenological scaling relationships can be more convenient in engineering and scientific practice. For example, it was established in [25] that in the case of a chemical underwater explosion, the peak pressure in the radiated impulse is a function of the charge mass, $M$, and the distance between the charge and the observation point, $\ell$ :

$$
P_{\max } \propto\left(M^{1 / 3} / \ell\right)^{\beta}
$$

where an empirical constant $\beta$ is found to be 1.13. As the energy provided to an explosively-driven system would be proportional to the mass of the explosive, it may be possible to adapt this phenomenological relationship to the case of a spark breakdown in water. It can be assumed that the charge mass, $M$, is proportional to the energy stored in the capacitor(s) of the pulsed-power system:

$$
M \propto C V_{b r}^{2} / 2
$$

Thus, by combining (10) and (11), the peak acoustic pressure in the first acoustic impulse radiated by the plasma cavity at any known distance from the spark discharge $(\ell=$ constant) would be expected to be proportional to the product of the breakdown voltage to the power $2 \beta / 3$, and to the capacitance to the power $\beta / 3$ :

$$
P_{\max } \propto C^{\beta / 3} V_{b r}^{2 \beta / 3}
$$

For a known value of capacitance, a coefficient of proportionality, $k$, between $P_{\max }$ and $V_{b r}$ can be introduced:

$$
P_{\max }=k V_{b r}^{2 \beta / 3}
$$



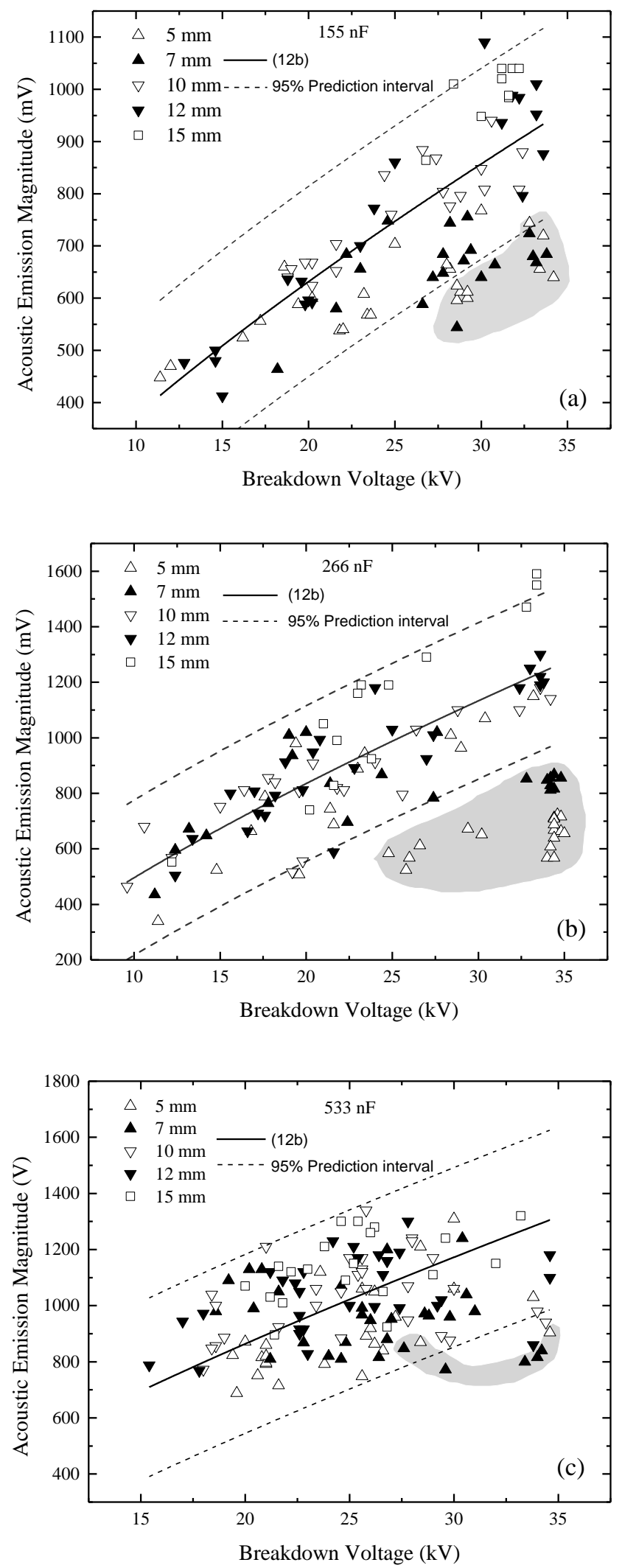

Fig. 5. Peak magnitude of the primary acoustic signal as a function of the breakdown voltage for (a) $155 \mathrm{nF}$, (b) $266 \mathrm{nF}$ and (c) $533 \mathrm{nF}$, with the fitting lines given by $(12 \mathrm{~b})$. The dashed lines show the upper and lower boundaries of the $95 \%$ prediction interval. The grey areas indicate those data points where $R_{\text {plasma }}<R_{\text {circuit. }}$
The maximum, peak pressure of the primary acoustic impulse emitted by the expanding cavity, $P_{\max }$, has been obtained experimentally, and plotted as a function of the breakdown voltage, $V_{b r}$, for all three capacitance values and for all five inter-electrode distances, Fig. 5. Each data point in Fig. 5 represents an individual measurement of the peak acoustic magnitude. The trends observed in Fig. 5 show that the maximum acoustic magnitude demonstrates the same functional behaviour as the period of cavity oscillations presented in Fig. 4. The peak pressure in the primary acoustic impulses increases with an increase in the breakdown voltage for all inter-electrode gaps. The solid lines in Fig. 5 represent the fitting lines obtained using (12b), with $\beta=1.13$ for the main increasing trends in the peak pressure. The coefficient of proportionality, $k$, was a free parameter in this fitting procedure, which was performed using Origin Pro 9 software package. Numerical values of $k$ (for the peak magnitude expressed in $\mathrm{mV}$ and the breakdown voltage expressed in $\mathrm{kV}$ ) are given in TABLE II.

TABLE II

FITTING PARAMETERS $k$ FOR $(12 \mathrm{~b})$

\begin{tabular}{c|c|c|c}
\hline \hline$C$ & $155 \mathrm{nF}$ & $266 \mathrm{nF}$ & $533 \mathrm{nF}$ \\
$k$ & 66.18 & 87.44 & 90.45 \\
95\% confidence & $(64.79-67.57)$ & $(84.98-89.89)$ & $(88.33-92.57)$ \\
interval & & & \\
\hline \hline
\end{tabular}

As in the case of the period of cavity oscillations, there are data points for breakdown of the shorter gaps $(5 \mathrm{~mm}$ and $7 \mathrm{~mm}$ ) and for voltages higher than $\sim 20 \mathrm{kV}$ which have lower peak pressure than the points within the $95 \%$ prediction intervals. This data, denoted by the grey areas in Fig. 5, is again associated with breakdowns were the plasma resistance is lower than the resistance of the driving circuit, $R_{\text {plasma }}<R_{\text {circuit }}$, and the lower acoustic peak magnitudes can again be explained by a decrease in the proportion of the electrical energy which is converted into thermal plasma energy. It is also clear from the results shown in Fig. 5 that the peak acoustic magnitude is higher for higher values of energy-storage capacitance, at the same breakdown voltages.

The coefficient $k$ in (12b) increases with an increase in the capacitance of the pulsed-power system. According to (12a) and (12b), $k \propto C^{0.37}$ - non-linear allometric fitting of the data in TABLE II gives a similar exponent value for $C, \sim 0.3$. This suggests that the scaling relation (12b) could be used for analysis of the functional behaviour of the acoustic magnitude. There is a significant spread in the peak acoustic magnitude as shown in Fig. 5, so (12b) should be used with a degree of caution, since it provides only the expected average value of the peak acoustic power. Nevertheless, this phenomenological scaling relation, which is based on (10), allows identification of the functional behaviour of $P_{\max }$, which makes this relation a valuable tool in practical engineering and scientific applications.

\section{3) Plasma resistance and dependency of the hydrodynamic parameters of cavity on $R_{\text {plasma }}$}

The resistance of the plasma channel, $R_{\text {plasma }}$, is an important factor in the optimisation of plasma-acoustic systems. $R_{\text {plasma }}$ defines the efficiency of conversion of the available electrical 
energy into thermal plasma energy and, therefore, determines the hydrodynamic parameters of the plasma cavity and the characteristics of the acoustic impulses emitted by this cavity. Although the actual plasma resistance is a time-varying parameter, it changes very rapidly and in a time interval which is shorter than a quarter of the period of the current oscillation, the plasma resistance reaches its minimum value [26]. As discussed in Section II, the constant-resistance model has been selected in the present work for obtaining an effective resistance for the plasma cavities and the energy dissipated in the plasma channels. The resistances of the pulsed-power driving circuit, $R_{\text {circuit }}$, with three different capacitances were obtained by extracting $\alpha$ and $\omega$ values from the experimental current waveforms and solving (1)-(2). The plasma-generating electrodes were short-circuited in this case, and the voltage controlled so that the peak current magnitudes were similar to those observed during water breakdowns. Average values of these resistances, obtained using at least 4 current waveforms, together with the corresponding standard deviations are listed in TABLE III. The same methodology was used to obtain the total resistance of the circuit, $R_{\text {total }}$, including the resistance of the plasma cavities, $R_{\text {plasma }}$, from the waveforms measured for each breakdown event. Equation (3) was then used to calculate the effective resistance of the plasma channel for each breakdown event.

TABLE III

\begin{tabular}{ccc}
\multicolumn{3}{c}{$R_{\text {circuit }}$ FOR DIFFERENT CIRCUIT CAPACITANCES, $C$} \\
\hline \hline$C(\mathrm{nF})$ & $R_{\text {circuit }}(\mathrm{m} \Omega)$ & $\begin{array}{c}\text { Standard deviation } \\
(\mathrm{m} \Omega)\end{array}$ \\
& & 20 \\
155 & 534 & 20 \\
266 & 401 & 7 \\
\hline 533 & 273 & \\
\hline \hline
\end{tabular}

In Fig. 6, the period of cavity oscillations is plotted as a function of the calculated effective plasma resistance for the three values of the circuit capacitance, and for all five inter-electrode gaps. Each data point in Fig. 6 represents an individual measurement of the peak acoustic magnitude. The vertical lines in each graph show the corresponding values of the circuit resistance, $R_{\text {circuit }}$.

The effective plasma resistance values vary in a wide range, from $35 \mathrm{~m} \Omega$ to $5.9 \Omega$. The plasma resistance generally increases with an increase in the inter-electrode gap length, as the length of the plasma channel also increases. Lower values of the effective plasma resistance can be attributed to higher energy levels delivered in the discharge and vice versa, lower energy levels result in lower values of the plasma resistance. Fig. 6 shows that the period of cavity oscillations initially increases with an increase in the plasma resistance, before reaching a maximum and then decreasing. This trend can be observed for all three values of capacitance in the pulsed-power circuit. The higher values of the plasma resistance have been registered in the case of $266 \mathrm{nF}$ capacitance: for this capacitance, breakdown of longer gaps was achieved using a charging voltage of $30 \mathrm{kV}$, which was not possible in the case of $155 \mathrm{nF}$ capacitance (where $35 \mathrm{kV}$ charging voltage was required). Thus, such combination of the circuit parameters resulted in a longer "tail" in the period of cavity oscillation as a function of the plasma resistance.
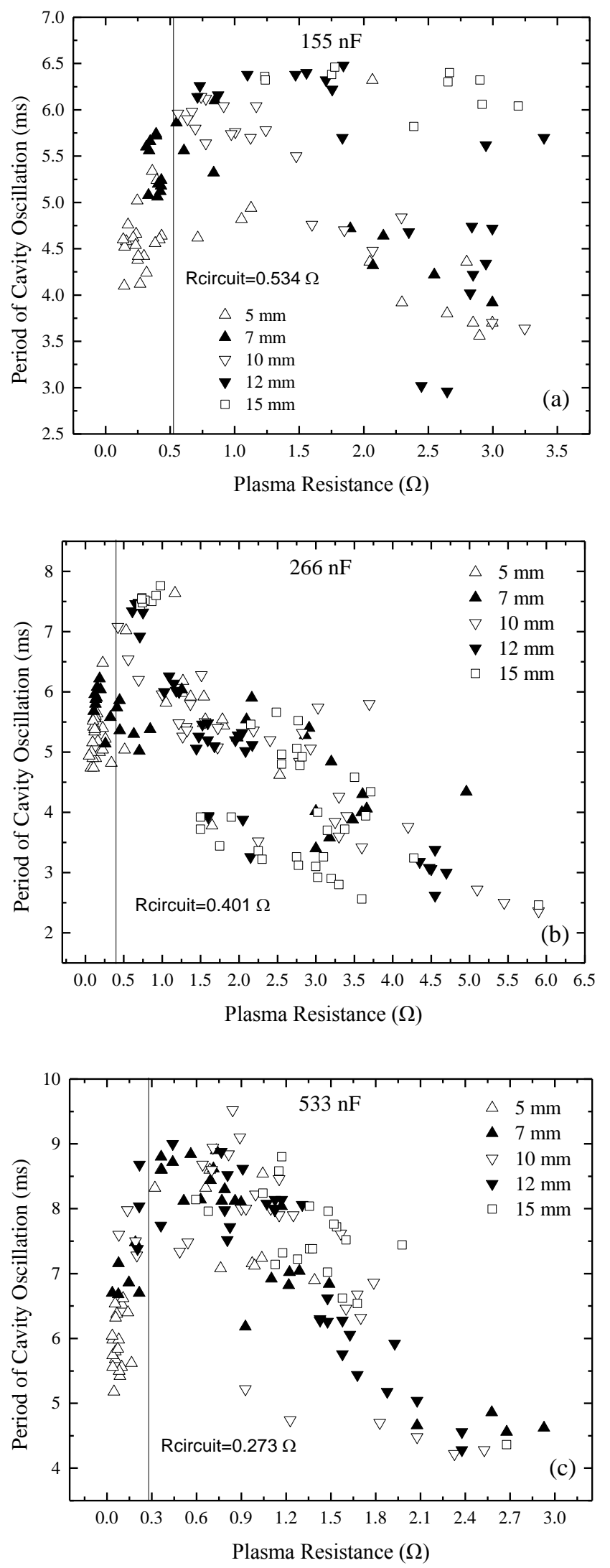

Fig. 6. Period of cavity oscillations as a function of the plasma resistance for (a) $155 \mathrm{nF}$, (b) $266 \mathrm{nF}$ and (c) $533 \mathrm{nF}$. The straight vertical lines represent the calculated resistances of the pulsed-power driving circuits. 
Theoretically, optimal energy transfer occurs when the resistance of the load is equal to that of the external driving circuit. However, Fig. 6 shows that the maximum oscillation periods are achieved for plasma resistances, $R_{\text {plasma }}$, greater than the circuit resistances, $R_{\text {circuit }}$. Calculated values of $R_{\text {circuit }}$ are shown by the vertical lines in Fig. 6, and it can be seen that the maximum values of the period of cavity oscillations are achieved at plasma resistances which are approximately twice as high as the pulsed-power circuit resistance: $\sim 1 \Omega$ for $155 \mathrm{nF}$; $\sim 0.8 \Omega$ for $266 \mathrm{nF}$; and $\sim 0.6 \Omega$ for $533 \mathrm{nF}$. The period of cavity oscillations reduces when the plasma resistance becomes lower or higher than these optimal values, this reduction being due to the smaller proportion of energy delivered into the plasma during the transient process, as compared with the total energy accumulated in the capacitor bank of the pulsed-power system. This reduction in the proportion of energy delivered into the plasma is also a possible reason for the saturation in the period of cavity oscillations for $5 \mathrm{~mm}$ and $7 \mathrm{~mm}$ gaps observed in Fig. 4 and Fig. 5.

Fig. 7 shows the relationship between the peak acoustic magnitude and the plasma resistance for all three values of the capacitance and for all five inter-electrode distances. As can be taken from Fig. 7, the functional behaviour of the peak acoustic magnitude for $155 \mathrm{nF}$ and $266 \mathrm{nF}$ capacitances is similar to that of the period of plasma oscillations shown in Fig. 6.

The peak acoustic magnitudes also tend to increase with an increase in the plasma resistance, and reach their maximum values at plasma resistances which are close to, but higher than, the calculated resistances of the pulsed-power circuit. However, the peak magnitudes behave more stochastically and do not show clear maxima at specific plasma resistances - only in the case of $266 \mathrm{nF}$ is it reasonable to state that the optimal resistance is $\sim 0.8 \Omega$. The functional behaviour of the peak acoustic magnitude in the case of $533 \mathrm{nF}$ capacitance is not well defined: although the peak magnitude initially increases with increasing resistance and reduces at higher values of resistance, it is difficult to identify the optimal value of $R_{\text {plasma }}$ for all tested inter-electrode gaps. For example, the majority of the peak acoustic magnitudes for the $5 \mathrm{~mm}$ gap lie along a horizontal line, which confirms saturation of the acoustic signal magnitude at higher energy levels.

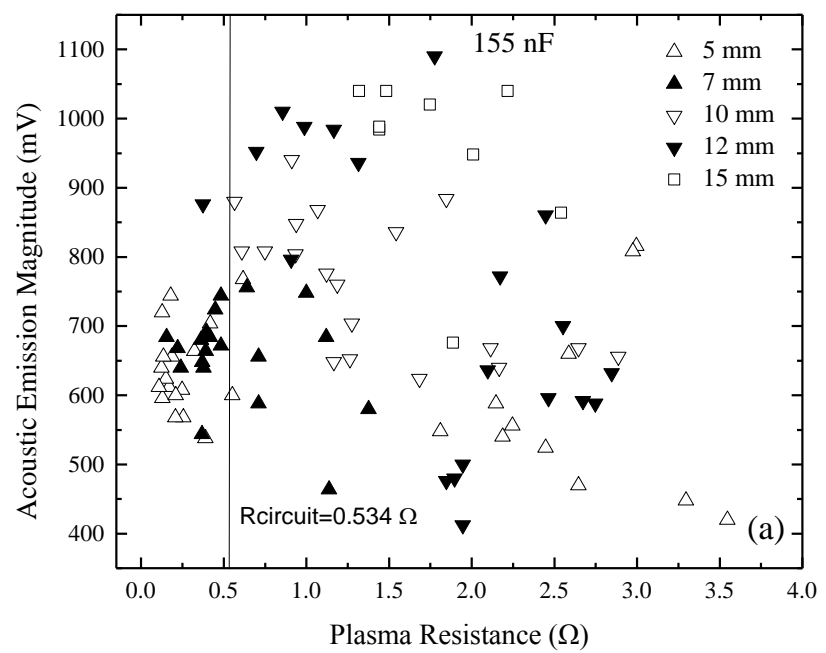

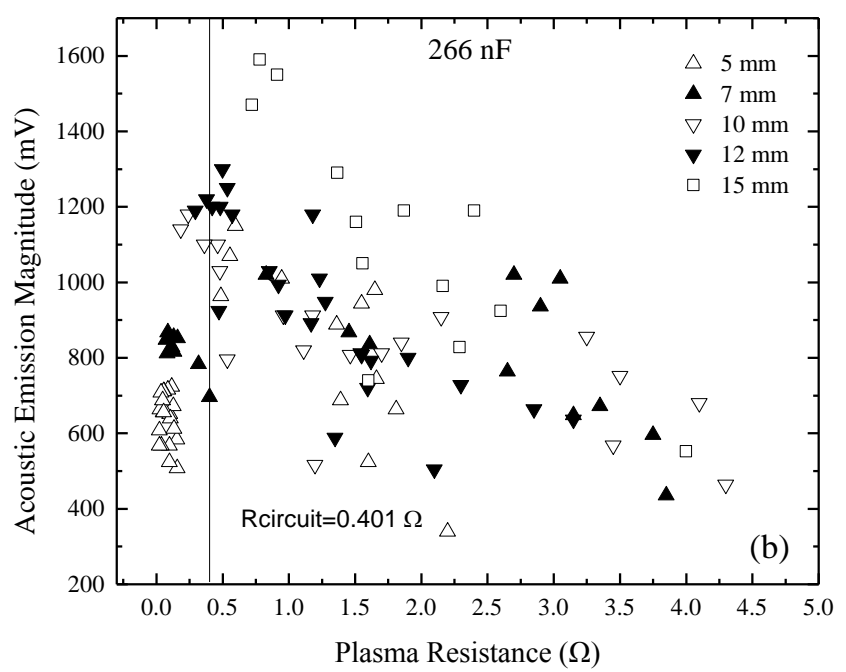

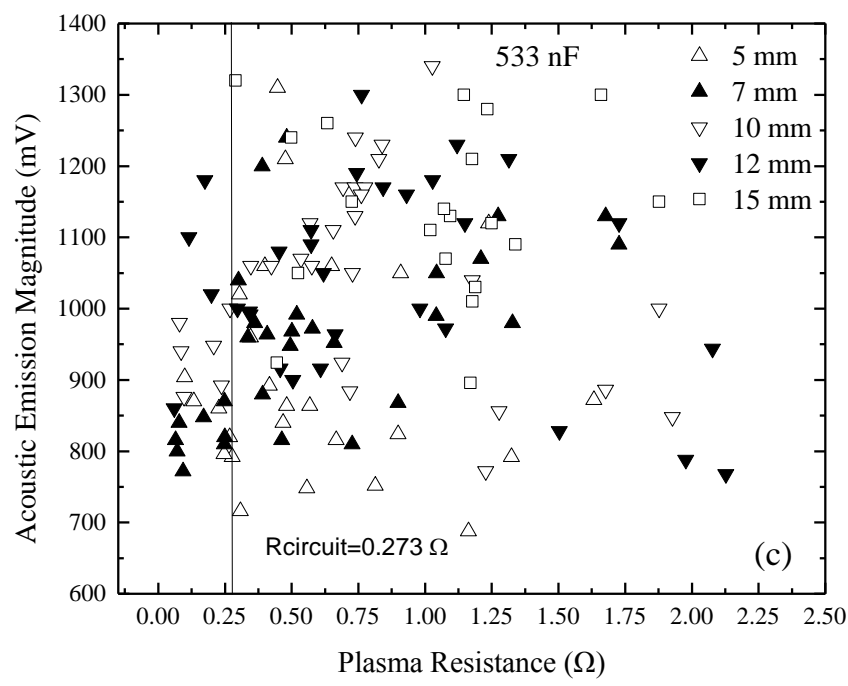

Fig. 7. Peak magnitude in the primary acoustic signal as a function of the plasma resistance for (a) $155 \mathrm{nF}$, (b) $266 \mathrm{nF}$, and (c) $533 \mathrm{nF}$. The straight lines represent the resistance of the pulsed-power circuit, $R_{\text {circuit }}$.

4) Energy dependency of hydrodynamic and acoustic parameters of the cavity

In Section III-B 2), the period of cavity oscillations and the peak acoustic pressure were obtained and presented as functions of the breakdown voltage. The phenomenological scaling relations established in Section III-B 2) are based on measurable or known circuit parameters, $V_{b r}$ and $C$, which make them useful for practical applications where the breakdown voltage is one of the main operating factors. However, in Section III-B 3), the plasma resistance associated with the breakdown channel was also obtained, which allows calculation of the energy delivered into the plasma channel to be made. Thus, it becomes possible to find a relationship between the energy dissipated in the plasma channel (eliminating pre-breakdown energy losses and losses in the pulsed-power circuit) and the hydrodynamic and acoustic parameters of the cavity. 
In the present section, an analysis of the behaviour of the period of cavity oscillations and the peak acoustic magnitude as functions of the energy delivered into the plasma channel, $W_{c h}$, is presented. $W_{c h}$ was obtained by integration of the product of the calculated constant plasma resistance, $R_{\text {plasma }}$, and squared values of the experimentally-measured current:

$$
W_{c h}=R_{\text {plasma }} \int I^{2}(t) d t
$$

Using (6), the following scaling relationship between the period of oscillations, $T_{\text {cavity }}$, and the energy delivered into the plasma channel was obtained:

$$
T_{\text {cavity }} \propto \gamma \rho^{1 / 2} p_{\infty}^{-5 / 6} W_{c h}^{1 / 3}
$$

For known values of $\gamma, \rho$ and $p$, a coefficient of proportionality, $n$, between $T_{\text {cavity }}$ and $W_{c h}$ can be introduced:

$$
T_{\text {cavity }}=n W_{c h}^{1 / 3}
$$

Fig. 8 shows the period of oscillations, $T_{\text {cavity }}$, as a function of $W_{c h}$, the energy delivered into the plasma channel, calculated using (13). Equation (14b) was used for fitting the experimental data points in Fig. 8. The proportionality coefficient, $n$, was a free parameter in the fitting procedure, which was conducted using Origin Pro 9 graphing software. In (14b), $T_{\text {cavity }}$ is in ms and $W_{c h}$ is in J. The fitting parameter $n$ and its $95 \%$ confidence intervals are given in TABLE IV.

TABLE IV

FITTING COEFFICIENT $n$ FOR SCALING RELATION (14b)

\begin{tabular}{cccc}
\hline \hline Capacitance & $155 \mathrm{nF}$ & $266 \mathrm{nF}$ & $553 \mathrm{nF}$ \\
$n$ & 1.69 & 1.68 & 1.59 \\
$95 \%$ confidence & $(1.67-1.72)$ & $(1.66-1.69)$ & $(1.58-1.62)$ \\
interval & & & \\
\hline \hline
\end{tabular}

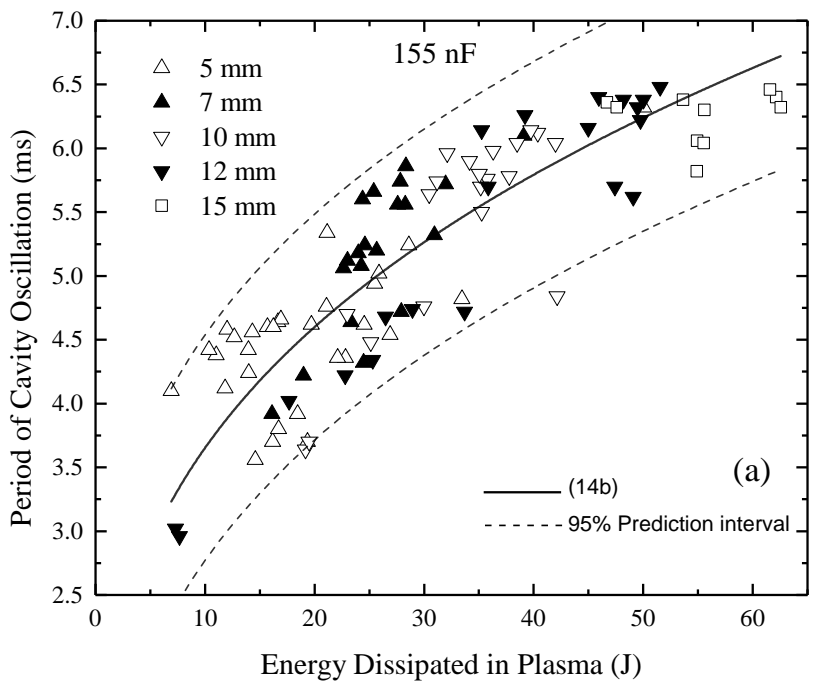

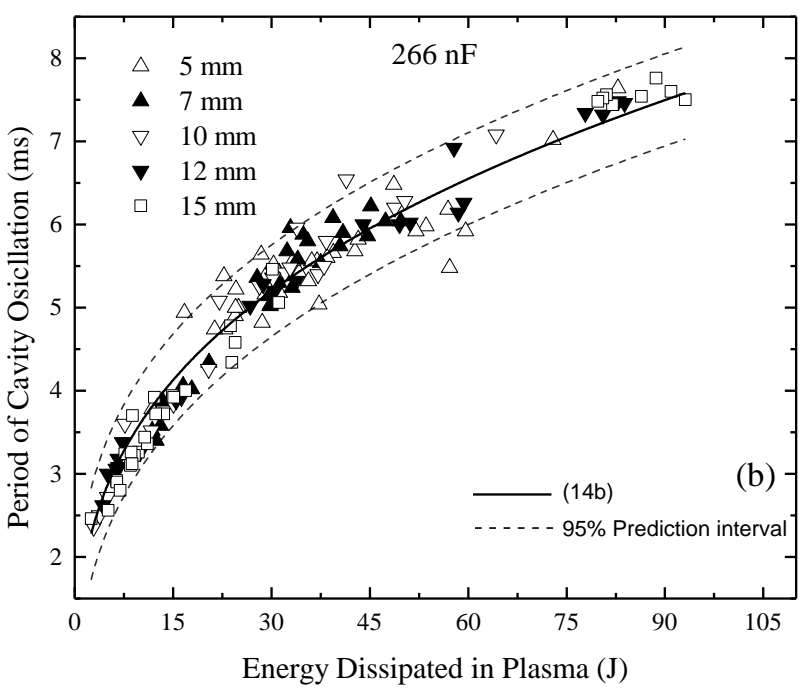

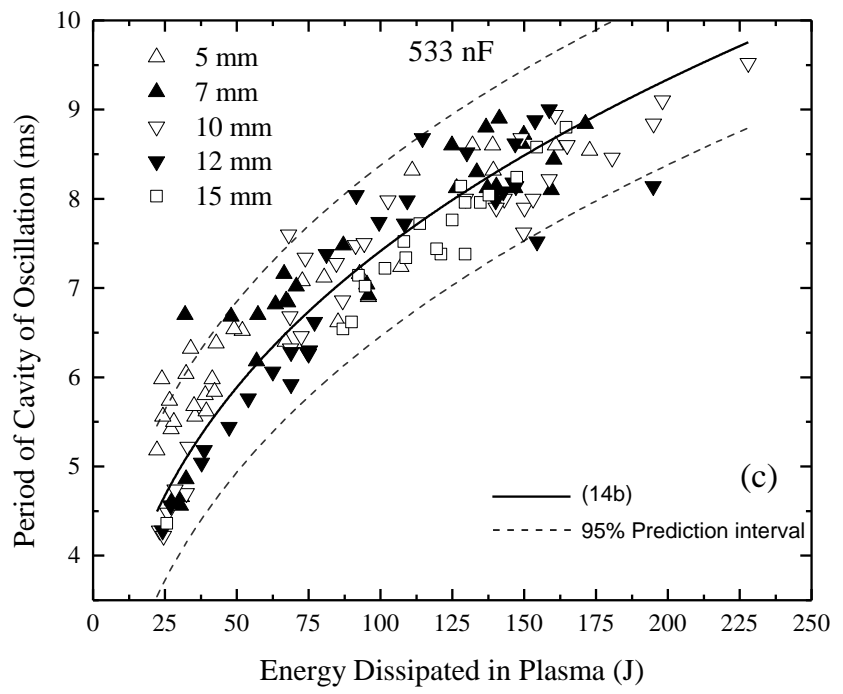

Fig. 8. Period of cavity oscillations as a function of the energy dissipated in the plasma channel for (a) $155 \mathrm{nF}$, (b) $266 \mathrm{nF}$ and (c) $533 \mathrm{nF}$, with the fitting curves given by (14b). The dashed lines show the upper and lower boundaries of the $95 \%$ prediction interval.

The quality of the fits of (14b) to the experimental data shows that this phenomenological scaling relation reasonably describes the functional behaviour of the period of cavity oscillation, and can be used for calculation of the expected values of $T_{\text {cavity. }}$.

In Section III-B 2), the functional dependency of the acoustic peak magnitude on the breakdown voltage was established. This scaling relation is helpful in practical applications in which the breakdown voltage can be readily obtained. However, if the resistance of the plasma channel is known and the discharge current waveform is available, the energy delivered into the plasma channel can also be calculated. It is important to investigate the relationship between this energy, $W_{c h}$, and the peak acoustic magnitude. The cumulative result of this analysis is shown in Fig. 9. This figure provides a cumulative graph, the natural logarithm of the peak acoustic magnitude as a function of the natural logarithm of the energy, $W_{c h}$, for all 3 capacitances and 5 inter-electrode distances. 


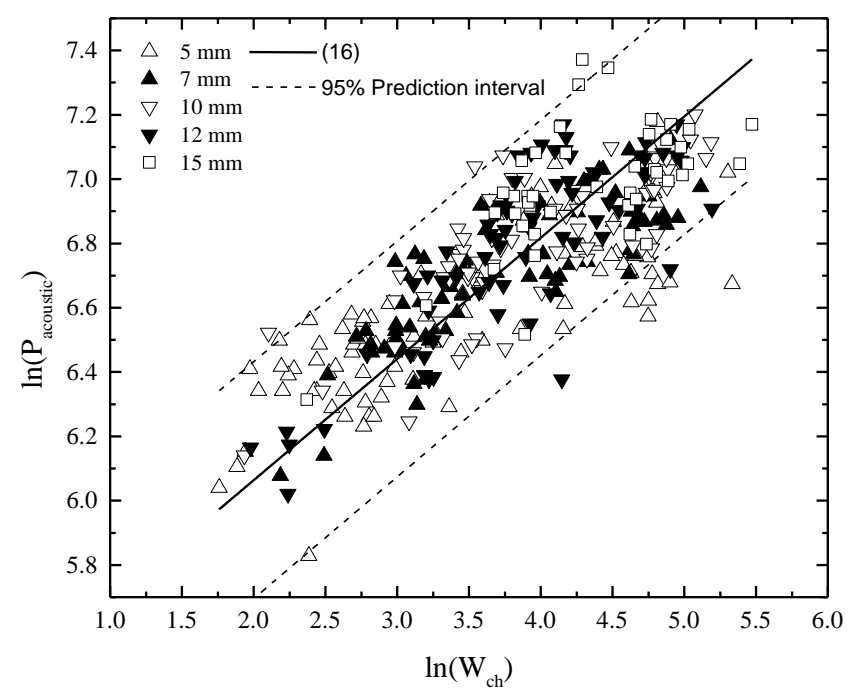

Fig. 9. Peak acoustic magnitude versus energy dissipated in the plasma channel, with the (solid) fitting line given by (16). The dashed lines show the upper and lower boundaries of the $95 \%$ prediction interval.

The phenomenological relation for the acoustic magnitude, $P_{\text {acoustic }}$, was obtained using (10) and assuming that $W_{c h} \propto M$ :

$$
P_{\text {acoustic }}=q W_{c h}^{\beta / 3}
$$

where $q$ is a coefficient of proportionality between $P_{\text {acoustic }}$ (expressed in $\mathrm{mV}$ ) and $W_{c h}$ (expressed in J). Taking the natural logarithm of both sides of (15), one can obtain:

$$
\ln \left(P_{\text {acoustic }}\right)=\ln (q)+(\beta / 3) \ln \left(W_{c h}\right)
$$

The solid line in Fig. 9 is a fitting line obtained by (16). The coefficient, $\ln (q)$, was a free parameter in this fitting procedure, and the power factor, $\beta$, was kept constant, $\beta=1.13$. It can be seen that (15) and (16) describe well the functional behaviour of the peak acoustic magnitude, and thus this phenomenological scaling relation can be used in practical applications such as the development of underwater plasma-acoustic sources.

It is worthy of note that for lower discharge energies, [27] and [28] provide similar analytical relations between the peak acoustic magnitude and the discharge energy: $P_{\max } \sim\left(W_{c h}{ }^{b} / p\right)$, where $p$ is the external/hydrostatic pressure and $b$ is a fitting constant which is close to the value of $\beta / 3$ used in this study. As in the case of (12), scaling relation (15) cannot be used for exact calculation of the peak acoustic magnitude, however this scaling relation helps to establish the functional behaviour of the peak magnitude, and can be used for prediction and evaluation of this magnitude as a function of the energy delivered into the underwater spark. Using (5) and (7), it is possible to evaluate the proportion of the electrical energy, $\gamma$, which is converted into the thermal plasma energy spent on cavity expansion. The values of $\gamma$ have been obtained by plotting $W_{c h}$ as a function of $R_{\text {circuit }}$ and using the fitting procedure in Origin Pro 9 software. It was found that $\gamma$ for all tested capacitances is $\sim 0.3$; these values, together with their 95\% prediction interval limits, are listed in TABLE V.

TABLE V

COEFFICIENT $\gamma$ FOR DIFFERENT CAPACITANCES

\begin{tabular}{ccc}
\hline \hline Capacitance & $\gamma$ & $\begin{array}{c}95 \% \text { confidence } \\
\text { interval }\end{array}$ \\
\hline $155 \mathrm{nF}$ & 0.32 & $(0.29-0.35)$ \\
$266 \mathrm{nF}$ & 0.35 & $(0.34-0.36)$ \\
$553 \mathrm{nF}$ & 0.25 & $(0.23-0.27)$ \\
\hline \hline
\end{tabular}

\section{CONCLUSIONS}

In this study, the functional behaviour of the hydrodynamic, electrical and acoustic parameters of underwater spark discharges has been investigated. The period of cavity oscillations, the peak magnitude of the acoustic impulses generated, and the resistance of the underwater plasma channels have been obtained as functions of the breakdown voltage, energy, circuit capacitance and inter-electrode distance.

It was shown that the period of cavity oscillations and the acoustic impulse magnitude increase with an increase in the breakdown voltage for discharges in all tested gaps. This increase can be described by the phenomenological scaling relations (9b) and (12b). However, for shorter gaps (5 mm and $7 \mathrm{~mm}$ ) and higher breakdown voltages ( $20 \mathrm{kV}$ and above), there are clusters of data points which deviate from the main trend lines. These data are located below the lower boundary lines for the $95 \%$ prediction intervals of the main trends. It was found that the plasma resistance for all data points located in these outlying clusters is lower than the circuit resistance. Therefore, this deviation of $T_{\text {cavity }}$ and $P_{\max }$ from the main trends can be explained by the less-efficient electrical to thermal energy conversion for these discharges.

The resistance of underwater plasma channels was obtained using the constant-resistance approach. The plasma resistance was found to vary from $\sim 0.035 \Omega$ to $\sim 5.9 \Omega$ for different energisation conditions and for different inter-electrode gaps. It was found that the period of cavity oscillations and the peak acoustic magnitude are non-linear functions of the plasma resistance; these values have their maxima when the resistance of the plasma is approximately twice higher than the resistance of the pulsed-power driving circuit.

The energy dissipated in the plasma channel was calculated using the experimental current waveforms and calculated constant plasma resistances. The period of cavity oscillations and the peak acoustic magnitude demonstrated a non-linear increase with an increase in the energy delivered into the plasma channel, and their functional behaviour is described by the phenomenological scaling relations (14b) and (15).

The results of this study will help in further understanding of the process of development of transient spark discharges in water, generation of acoustic impulses by such discharges, and in optimisation of plasma-acoustic sources, through tailoring of the pulsed-power circuit parameters to specific applications. 


\section{REFERENCES}

[1] W. Stygar, M. Savage, T. Wagoner et al, "Dielectric-break down tests of water at $6 \mathrm{MV}$ ", Physical Review Special Topics-Accelerators and Beams, vol. 12, 01402, pp. 1-10, 2009.

[2] C. Chaussy, E. Schmiedt, "Extracorporeal shock wave lithotripsy (ESWL) for kidney stones, An alternative surgery?", Urol. Radiol., vol. 6, no. 1, pp. 60-67, 1984.

[3] M. Wilson, L. Balmer, M. Given, S. MacGregor,

I. Timoshkin, "An investigation of spark discharge parameters for material processing with high power ultrasound", Minerals Engineering, vol. 20, pp. 1159-1169, 2007.

[4] W. Carley-Macauly, J. Hitchen, N. Maroudas, "Energy consumption in electrohydraulic crushing", Trans. Inst. Chem. Eng., vol. 44a, pp. T395-T404, 1966.

[5] K. Carley-Macauly, "Electrohydraulic crushing", Chem. And Proc. Eng., vol. 49, no. 9, pp. 87-92, 1968.

[6] M. Wilson, L. Balmer, M. Given, S. MacGregor,

J.Mackersie and I. Timoshkin, "Apploations of electric spark generated high power ultrasound to recover ferrous and non-ferrous metals from slag waste", Minerals Engineering, vol. 20, pp. 1159-1169, 2007.

[7] K. Chung S. Lee, Y. Hwang, C. Kim, "Modelling of pulsed spark discharge in water and its applications to well cleaning", Current Applied Phys., vol. 15, pp 977-986, 2015.

[8] E. Martin, "Experimental investigation of a high-energy, high-pressure arc plasma", J. of Appl. Phys., vol. 31, pp. 255-267, 1960.

[9] S. Buogo, G. Canneli, "Implosion of an underwater spark-generated bubble and acoustic energy evaluation using the Rayleigh model", J. Acout. Soc. Am., vol. 111, no. 6, pp. 2594-2600, 2002.

[10] V. Atrazhev, V. Vorob'ev, I. Timoshkin, M. Given,

S. MacGregor "Mechanisms of impulse breakdown in liquid: the role of joule heating and formation of gas cavities", IEEE Trans. on Plasma Sci., vol. 38, no. 10, pp. 2644-265, 2010.

[11] T. J. Lewis, "New electro-mechanical concepts for the primary mechanism of electrical breakdown in liquids", $12^{\text {th }}$ Int. Conf. on Conduction and Breakdown in Dielectric Liquids, Rome, Italy, pp. 272-278, 1996.

[12] B. S. Sommers, J.E. Foster, N.Y. Babaeva, M.J. Kushner, "Observations of electric discharge streamer propagation and capillary oscillations on the surface of air bubbles in water", $J$. Appl. Phys D: Applied Physics, vol. 44, 082001, pp. 1-6, 2011. [13] J. Qian, P. Joshi, K. Schoenbach, G. Sarkisov, "Model analysis of self- and laser triggered electrical breakdown of liquid water for pulsed power applications", IEEE Trans. On Plasma Science, vol.34, no.5, pp.1680-1691, 2006.

[14] R. Joshi, S. Thagard, "Streamer-like electrical discharges in water: part I. fundamental mechanisms", Plasma Chem. Process., vol.33, pp.1-15, 2013.

[15] V. Y. Ushakov, V. Y. Klimkin, S. M. Korobeynikov, Impulsive Breakdown of Liquids. Berlin, Germany: Springer-Verlag, 2007.

[16] M. Shneider, "Dielectric fluid in inhomogeneous pulsed electric field", Phys. Rev., E87, 043004, 2013.

[17] Y. Seepersad, M. Pekker, M. Shneider, D. Dobrynin, “On the electrostrictive mechanism of nanosecond-pulsed breakdown in liquid phase", J. Phys. D: Appl. Phys., vol.46, 162001, pp. 1-6, 2013.

[18] I. Timoshkin, R. Fouracre, M. Given, S. MacGregor, "Hydarodynamic modeling of transient cavities in fluids generated by high voltage spark discharges", J. Phys. D: Appl. Phys., vol. 39, pp. 4808-4817, 2006

[19] J. W. Mackersie, I. V. Timoshkin and S. J. MacGregor, "Generation of high-power ultrasound by spark discharge in water", IEEE Trans. on Plasma Sci., vol. 33, no. 5, pp. 1715-1724, 2005.

[20] J. Cook, A. Gleeson, R. Roberts, "A spark-generated bubble model with semi-empirical mass transport", J. Acoust. Soc. Am., vol.101, no.4, 1908-1920, 1997.

[21] K. A. Naugol'nykh and N. A. Roi, "Spark discharges in water (a hydrodynamical description)", (Translation, Foreign Technology Division), Wright-Patterson AFB, Dayton, $\mathrm{OH}$, USA, Tech. Rep., 1974.

[22] J. P. VanDevender, "The resistive phase of a high-voltage water spark”, J.Appl.Phys., vol. 49, pp 2616-2620, June, 1978. [23] J. M. Lehr et al, "Multi-megavolt switching in water: considerations for the Z-R machine", in Proc. Int. Conf. on Electrical Insulation and Dielectric Phenomenon, Cancun, Mexico, pp. 554-557, 2002.

[24] T. G. Leighton, "The Acoustic Bubble", Academic Press, 1997.

[25] J. R. McGrath, "Scaling Underwater Exploding Wires", NRL Report 6266, Energy Conversion Branch, Electronic Division, US Naval Research Laboratory, Washington, 1965.

[26] K. B. Cunningham, A. J. Coleman, T. G. Leighton and P. R. White, "Characterizing in vivo acoustic cavitation during lithotripsy with time-frequency methods", Acoust. Bull., vol. 26, no. 5, pp 10-16, 2001.

[27] G. Touya, T. Reess, L. Pecastaing, A. Gibert, and P. Domens, "Development of subsonic electrical discharges in water and measurements of the associated pressure waves," J. Phys. D: Appl. Phys., vol. 39, no. 24, pp. 5236-5244, 2006. [28] J. Martin, "Etude et caractérisation d'onde de pression générée par une décharge électrique dans l'eau," Ph.D. thèse, à l'Université de Pau et des Pays de l'Adour, La faculté des sciences et techniques, Pyrénées-Atlantiques, France, 2013.

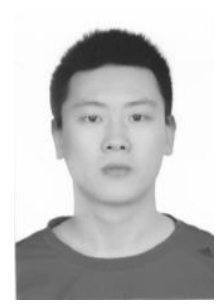

Ying Sun was born in Shandong, China. He received the B.Eng degree in electronic and electrical engineering in 2012 and M.Sc degree in electrical power engineering with business in 2013 from University of Strathclyde, Scotland, United Kingdom. He is currently pursuing the Ph.D degree in high voltage technology at University of Strathclyde, Scotland, United Kingdom.

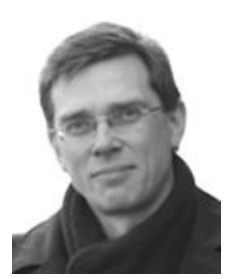

Igor V. Timoshkin (M'07-SM'14) received a degree in physics from the Moscow State University (Russia) in 1992, and the Diploma and the Ph.D. degree from the Imperial College of Science, Technology and Medicine, (London, UK) in 2001. After graduation from MSU he worked as a 
Researcher at Moscow State Agro-Engineering University, and then at the Institute for High Temperatures of Russian Academy of Sciences before moving to ICSTM in 1997. He joined the Department of Electronic and Electrical Engineering of the University of Strathclyde (Glasgow, UK) in 2001 where he became a Reader in 2016. His research interests include properties of solid and liquid dielectric materials, electronics of plasma discharges in condensed media, practical applications of electro-hydraulic and high-power ultrasound pulses, bio-dielectrics and effects of electromagnetic fields on biological objects.

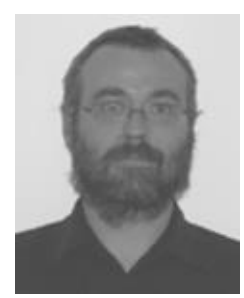

Martin J. Given (M'99-SM'11) is currently a Senior Lecturer in the Department of Electronic and Electrical Engineering at the University of Strathclyde. He received a degree in physics from the University of Sussex in 1981 and a PhD in electronic and electrical engineering from the University of Strathclyde in 1996. His research interests include, ageing processes and condition monitoring in solid and liquid insulation systems, high speed switching and pulse power.

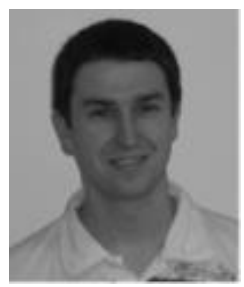

Mark P. Wilson (M'10) was born in Stranraer, Scotland, in 1982. He received the B.Eng. (with honours), M.Phil., and Ph.D. degrees in electronic and electrical engineering from the University of Strathclyde, Glasgow, U.K., in 2004, 2007, and 2011, respectively. $\mathrm{He}$ is presently working as a Teaching Associate at the University of Strathclyde, where he continues to investigate surface flashover of solids immersed in insulating oil. Mark is a member of the IEEE Nuclear and Plasma Sciences Society, from whom he received a Graduate Scholarship Award in 2011, the IEEE Dielectrics and Electrical Insulation Society, and the IET.

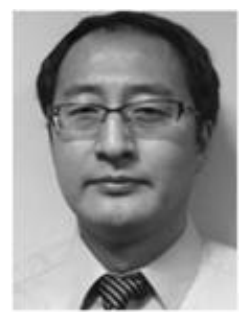

Tao Wang received the B.Eng and M.Sc degrees from Northeast China Dianli University (China) in 1993 and 1996 respectively, and the Ph.D. degree from the University of Strathclyde (Glasgow, UK) in 2005. He then joined the Newland Entech as a research fellow developing high efficiency industrial ozone generator. He joined the department of Electronic and Electrical Engineering of University of Strathclyde as a lecturer in 2010. His research interests include non-thermal gas discharges and their applications in gas synthesis, water disinfection and advanced oxidation process in water.

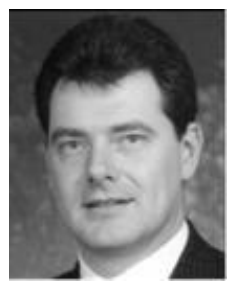

Scott J. MacGregor (M'95-SM'14) received the B.Sc. and Ph.D. degrees from the University of Strathclyde, Glasgow, U.K., in 1982 and 1986, respectively. He became a Pulsed Power Research Fellow in 1986 and a Lecturer in pulsed-power technology in 1989. In 1994, he became a Senior Lecturer, with a promotion to Reader and Professor of High Voltage Engineering, in 1999 and 2001, respectively. In 2006 and 2010 he became Head of the Department of Electronic and Electrical Engineering and Executive Dean of the Faculty of Engineering, and has been the Vice-Principal of the University of Strathclyde since 2014. Professor MacGregor was the recipient of the 2013 IEEE Peter Haas Award, and he was appointed as an Associated Editor of the IEEE Transitions on Dielectrics and Electrical Insulation in 2015. His research interests include high-voltage pulse generation, high-frequency diagnostics, high-power repetitive switching, high-speed switching, electronic methods for food pasteurization and sterilization, generation of high-power ultrasound (HPU), plasma channel drilling, pulsed-plasma cleaning of pipes, and stimulation of oil wells with HPU.

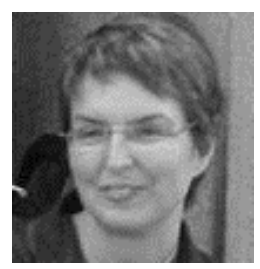

Nelly Bonifaci graduated from University Joseph Fourier with a MEng degree in Physics in 1989. In 2008 she obtained the HDR Habilitation qualification from the University of $\mathrm{J}$ Fourier, Grenoble. Dr Bonifaci joined the Laboratory on Dielectric Materials and Electrostatics (now G2E laboratory) CNRS, France in 1989 where she became a $1^{\text {st }}$ class researcher in 1997. In 2007 she was promoted to a position of a Senior Researcher/Team Leader. Dr Bonifaci research interests include breakdown in insulating liquids, plasma streamers in liquid dielectrics, pre-breakdown and plasma phenomena in dielectric liquids including corona discharges and electronic transport. Dr Bonifaci is an Editor of the IEEE Transactions of Dielectric and Electrical Insulation and a member of International Organising Committee of the IEEE Conference on Dielectric Liquids. 